\title{
Universiteit
}

Leiden

The Netherlands

\section{How to observe high-dimensional two-photon entanglement with only two detectors}

Oemrawsingh, S.S.R.; Aiello, A.; Eliel, E.R.; Nienhuis, G.; Woerdman, J.P.

\section{Citation}

Oemrawsingh, S. S. R., Aiello, A., Eliel, E. R., Nienhuis, G., \& Woerdman, J. P. (2004). How to observe high-dimensional two-photon entanglement with only two detectors. Physical Review Letters, 92(21), 217901. doi:10.1103/PhysRevLett.92.217901

Version: $\quad$ Not Applicable (or Unknown)

License: $\quad$ Leiden University Non-exclusive license

Downloaded from: https://hdl.handle.net/1887/65505

Note: To cite this publication please use the final published version (if applicable). 


\title{
How to Observe High-Dimensional Two-Photon Entanglement with Only Two Detectors
}

\author{
S. S. R. Oemrawsingh, A. Aiello, E. R. Eliel, G. Nienhuis, and J. P. Woerdman \\ Huygens Laboratory, Leiden University, P.O. Box 9504, 2300 RA Leiden, The Netherlands
}

(Received 2 February 2004; published 24 May 2004)

\begin{abstract}
We propose a novel setup to investigate the entanglement of orbital angular momentum states living in a high-dimensional Hilbert space. We incorporate noninteger spiral phase plates in spatial analyzers, enabling us to use only two detectors. The two-photon states that are produced are not confined to a $2 \times 2$-dimensional Hilbert space, and the setup allows the probing of correlations in a high-dimensional space. For the special case of half-integer spiral phase plates, we predict that the Clauser-HorneShimony-Holt-Bell parameter $S$ is larger than achievable for two qubits $\left(S=2 \sqrt{2}\right.$ ), namely, $S=3 \frac{1}{5}$.

DOI: 10.1103/PhysRevLett.92.217901

PACS numbers: 03.67.Mn, 42.50.Dv
\end{abstract}

Recently, the orbital angular momentum (OAM) of light has drawn considerable interest in the context of quantum information processing. The spatial degrees of freedom involved in OAM [1] provide a highdimensional alphabet to quantum information processing (i.e., qunits instead of qubits) [2,3]. Additionally, since OAM is associated with the topology of the electromagnetic field, the use of this observable in quantum entanglement may lead to states that are inherently robust against decoherence [4]. Popular methods used to prove the entanglement of the OAM degree of freedom of two photons (by showing that a generalized Bell inequality is violated) require six detectors, namely, three in each arm, and $3 \times 3$ coincidence count rates [3] to perform a measurement for a single setting of the analyzers.

In the present Letter, we consider the use of spiral phase plates (SPPs) [5], enabling us to investigate highdimensional entanglement with only two detectors. More specifically, we shall consider SPPs that impose on an optical beam a noninteger OAM expectation value per photon, in units of $\hbar$ [5]. Such devices, combined with single-mode fibers to form quantum-state analyzers, allow the observation of high-dimensional entanglement $(N>2)$, in contrast to the polarization case $(N=2)$. These claims are supported by quantitative calculations; we predict highly nonclassical quantum correlations $(S=$ $\left.3 \frac{1}{5}\right)$, i.e., stronger quantum correlation between two photons than the maximum correlation between two qubits $(S=2 \sqrt{2})$.

A SPP, shown in Fig. 1(a), is a transparent dielectric plate with a thickness that varies as a smooth ramp, thus phase shifting an incident field linearly with the azimuthal angle $\theta$ [5]. Placing such a plate in the waist of a Laguerre-Gaussian beam, the field in the exit plane just behind the plate will be described by

$$
\langle r, \theta|\hat{S}(\ell)| l, p\rangle=u_{\mathrm{LG}}^{l p}(r, \theta) \exp (i \ell \theta),
$$

where $|l, p\rangle$ are the Laguerre-Gaussian (LG) field states, $\hat{S}(\ell)$ is the operator representing the effect of the SPP on the input mode, and $\ell$ is the phase shift per unit angle.
The function $u_{\mathrm{LG}}^{l p}(r, \theta)=\langle r, \theta \mid l, p\rangle$ in Eq. (1) is the complex amplitude of the Laguerre-Gaussian beam in its waist plane [1,6]. Equation (1) describes a field amplitude that can be written as a superposition of an infinite number of LG components. When we require a certain fidelity, this number increases with $\ell$; as an example, for $\ell=\frac{1}{2}, 11 \mathrm{LG}$ components are sufficient to describe $87 \%$ of the field behind the SPP, while for $\ell=\frac{5}{2}, 224 \mathrm{LG}$ components are required.

From a topological point of view, SPPs with noninteger $\ell$ imprint a mixed screw-edge dislocation on an incident field. The result is rotational asymmetry of the imprinted phase distribution and thus of the emerging field, which becomes visible in the far-field intensity profile [Fig. 1(b)]. It is the orientation of the step in the transverse plane that we wish to exploit as an analyzer setting in a new bipartite entanglement scheme.

This proposed scheme is shown in Fig. 2; it has been inspired by the setup used in polarization entanglement [7]. We may orient the noninteger- $\ell$ SPPs arbitrarily in the transverse plane, as shown in Fig. 2, thus allowing their use as angular analyzers.

We stress that, in spite of the superficial similarity between a polarizer and a noninteger SPP, they are of course very different devices; for example, while polarization corresponds to alignment, the SPP edge corresponds to orientation. An equally important difference (a)

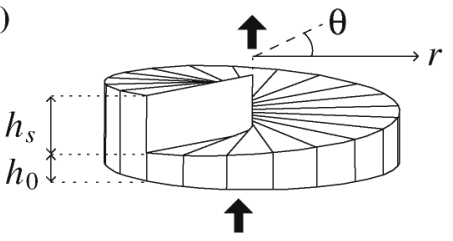

(b)

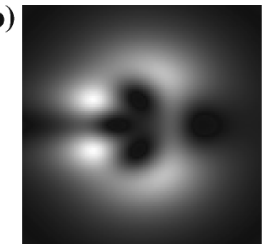

FIG. 1. (a) Schematic drawing of a SPP. The device shifts the phase of an incident beam proportional to the azimuthal angle $\theta$. (b) A calculated far-field diffraction pattern of a fundamental Gaussian beam after propagating through an $\ell=3 \frac{1}{2}$ plate positioned in its waist plane, showing that rotational symmetry is broken. Black and white denote low and high intensity, respectively. 


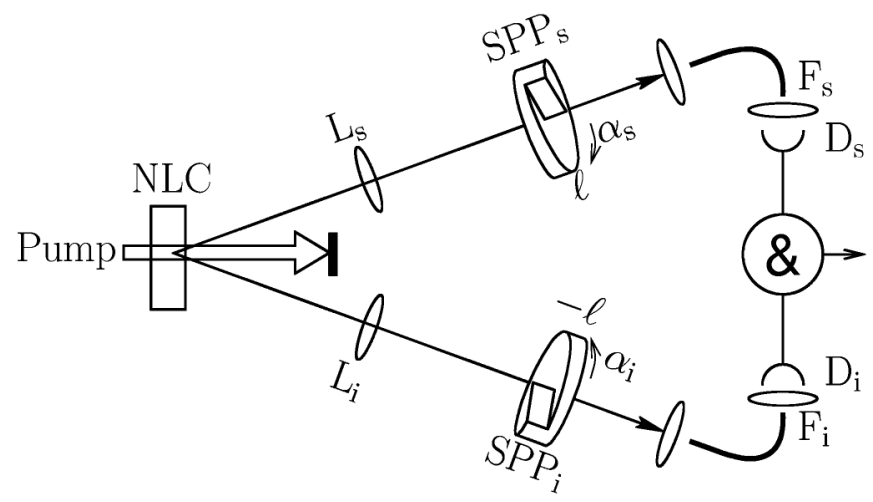

FIG. 2. Proposed experimental setup. A nonlinear crystal (NLC) splits a pump photon in a signal photon and an idler photon by the process of spontaneous parametric downconversion. In each path, a $\operatorname{SPP}\left(\mathrm{SPP}_{s, i}\right)$ is inserted with a single-mode fiber $\mathrm{F}_{s, i}$, together forming the analyzer. The coincidence count rate of detectors $\mathrm{D}_{s, i}$ is measured as a function of the SPP angular orientations $\alpha_{\mathrm{s}}$ and $\alpha_{\mathrm{i}}$.

between the two cases is that whereas polarization Hilbert space is two-dimensional, OAM Hilbert space is infinite dimensional. Moreover we assume that OAM is conserved in spontaneous parametric down-conversion processes. This issue has been discussed in several recent works $[2,8-13]$ and it seems that OAM is indeed conserved if two conditions are satisfied, namely, (i) the paraxial limit and (ii) the thin-crystal approximation. In practice, this is usually the case. In order to address all these aspects explicitly, we need a basis of the OAM Hilbert space that is suited for our purpose.

In the polar representation in real space, the LG-basis functions can be separated into a radial part $\rho_{l p}(r)$ and an azimuthal part $\phi_{l}(\theta)$ so that $\langle r, \theta \mid l, p\rangle=\rho_{l p}(r) \phi_{l}(\theta)$, where $\phi_{l}(\theta) \equiv e^{i l \theta} / \sqrt{2 \pi}$ are the eigenfunctions of the OAM operator, $\hat{\mathcal{L}}_{z}|l\rangle=l|l\rangle$; they provide a complete, orthonormal basis for the OAM Hilbert space.

We now transform the basis $\{|l\rangle\}$ from integer to noninteger OAM by applying a unitary operator, which ensures that completeness and orthonormality of the basis are conserved. The natural choice for this operator is the spiral phase plate operator $\hat{S}(\ell)$ introduced in Eq. (1). It is useful to generalize the definition of $\hat{S}(\ell)$ to $\hat{S}(\alpha, \ell)$ by including the orientation $\alpha$ of the edge dislocation. We define the new basis $\left\{\left|a_{\lambda}^{(l)}(\alpha)\right\rangle\right\}$ as $\hat{S}(\alpha, \ell)|l\rangle \equiv\left|a_{\lambda}^{(l+j)}(\alpha)\right\rangle$, where $j$ is the integer part of the step index $\ell$ (not to be confused with the integer LG index $l$ ) and

$$
\langle\hat{r}|\hat{S}(\alpha, \ell)| l\rangle=\frac{e^{i(l+\ell) \theta}}{\sqrt{2 \pi}} \times\left\{\begin{array}{cc}
e^{i(2 \pi-\alpha) \ell}, & 0 \leq \theta<\alpha \\
e^{-i \alpha \ell}, & \alpha \leq \theta<2 \pi
\end{array}\right.
$$

where $\alpha, \theta \in[0,2 \pi)$.

Let now $\alpha=0$; since the basis $\left\{\left|a_{\lambda}^{(l)}(0)\right\rangle\right\}$ is complete, the states after rotation, $\left|a_{\lambda}^{(l)}(\alpha)\right\rangle$ can be written as a superposition of these basis states. Thus the decomposition of $\left|a_{\lambda}^{(l)}(\alpha)\right\rangle$ into the basis $\left\{\left|a_{\lambda}^{(l)}(0)\right\rangle\right\}$ depends on the angle $\alpha$. To illustrate this, we make a projection of a noninteger state oriented at $\alpha=0$ onto the same state with orientation $\alpha \neq 0$. For this, we choose a SPP with step $\ell=j+\lambda$, where $\lambda \in[0,1)$, yielding the overlap amplitude $A(\alpha)=\left\langle a_{\lambda}^{(l+j)}(0) \mid a_{\lambda}^{(l+j)}(\alpha)\right\rangle$ and the probability

$$
|A(\alpha)|^{2}=\left(1-\frac{\alpha}{\pi}\right)^{2} \sin ^{2}(\lambda \pi)+\cos ^{2}(\lambda \pi)
$$

which depends neither on the integer part of the step index $j$ nor on the OAM state $l$. For nonzero values of $\lambda$, this overlap function has a quadratic dependence on the orientation $\alpha$. This function is plotted in Fig. 3 for various values of $\lambda$. It illustrates that, when $\lambda=0$, the projection does not change, as expected. For values of $\lambda \neq 0$, the outcome of the projection is less trivial, with $\lambda=\frac{1}{2}$ providing an especially interesting case: when the state is rotated over $\alpha=\pi$ by rotating the SPP, the state is orthogonal to the nonrotated state. To make our proposed OAM entanglement setup maximally equivalent to the polarizer setup, we focus from now on mainly on the case $\lambda=\frac{1}{2}$. Note that, as Eq. (3) is independent of $j$, any SPP with $\ell=j+\lambda$ may be used, as long as $\lambda=\frac{1}{2}$.

There is, however, a key difference between the halfinteger OAM states on the one hand and polarization states on the other hand. Angularly intermediate states of polarization are a superposition of two orthogonal basis states, say, horizontal and vertical linear polarization, so that the overlap function varies as $\sin ^{2} \alpha$. However, from Eq. (3) we see that in the present case the dependence of $|A|^{2}$ on $\alpha$ yields a parabola. Thus we conclude that, as the SPP is rotated the OAM state follows a path through Hilbert space that is not confined to a twodimensional subspace.

When choosing a fundamental Gaussian pump beam, described by the function $\langle\vec{r} \mid 0,0\rangle=\rho_{00}(r) / \sqrt{2 \pi}$, and

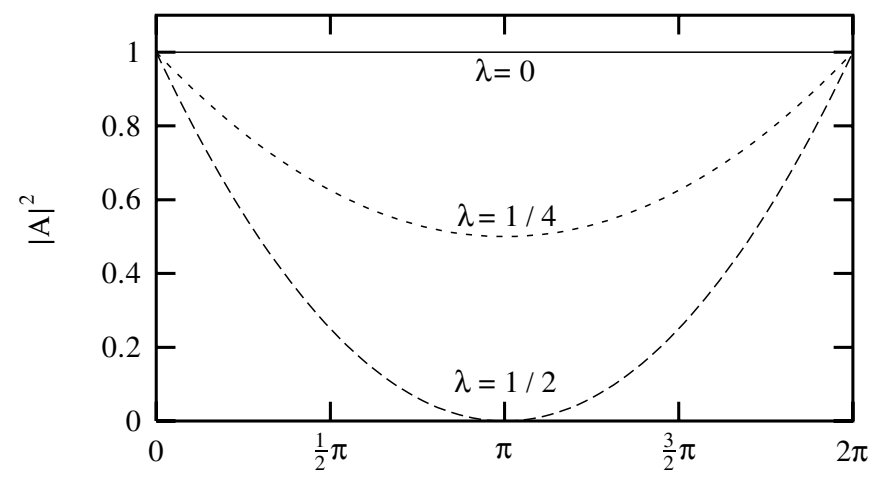

FIG. 3. The overlap [see Eq. (3)] between a noninteger OAM state and the identical state rotated over $\alpha$. When $\lambda=0$, the states are identical aside from a trivial phase shift, due to the vanishing edge dislocation. For half-integer OAM, i.e., $\lambda=\frac{1}{2}$, the two states are generally different, leading to a parabolic dependence of their overlap; the two states are orthogonal when $\alpha=\pi$. 
accounting for OAM conservation, the two-photon state can be described in the spatial polar representation as [14]

$$
|\Psi\rangle=\frac{1}{\sqrt{2 \pi}} \int \mathrm{d} \vec{r} \rho_{00}(r)|\vec{r}\rangle_{1}|\vec{r}\rangle_{2} .
$$

As discussed previously, we can separate the two-photon state into a radial and angular part, yielding

$$
|\Psi\rangle=\frac{1}{\sqrt{2 \pi}}|R\rangle \int_{0}^{2 \pi} \mathrm{d} \theta|\hat{r}\rangle_{1}|\hat{r}\rangle_{2},
$$

where we have defined the radial ket

$$
|R\rangle \equiv \int_{0}^{\infty} \mathrm{d} r r \rho_{00}(r)|r\rangle_{1}|r\rangle_{2},
$$

and the direction (i.e., angular) ket $|\hat{r}\rangle$. By applying a closure relation for both signal and idler photons in the half-integer SPP basis $\left\{\left|a_{1 / 2}^{(n)}(0)\right\rangle\right\}$ and subsequently removing the closure relation with respect to the angular coordinate, we can write the two-photon state as [15]

$$
|\Psi\rangle=\frac{1}{\sqrt{2 \pi}}|R\rangle \sum_{n=-\infty}^{\infty}\left|a_{1 / 2}^{(n)}(0)\right\rangle\left|a_{1 / 2}^{(-n-1)}(0)\right\rangle .
$$

We can now calculate the coincidence fringe that is expected in the proposed experiment (Fig. 2). In the signal path we place an analyzer consisting of a SPP with $\ell=$ $j+\frac{1}{2}$, where $j$ is an integer, with its orientation set to $\alpha=$ 0 , represented by $\hat{S}\left(0, j+\frac{1}{2}\right)$, and a single-mode fiber projecting on the LG state $|0,0\rangle \equiv\left|\rho_{00}\right\rangle|0\rangle$. When the detector clicks, the signal state before passing through this analyzer is collapsed to $\hat{S}^{\dagger}\left(0, j+\frac{1}{2}\right)|0,0\rangle \propto$ $\left|a_{1 / 2}^{(-j-1)}(0)\right\rangle$, resulting in a Kronecker delta $\delta_{j, n}$ in Eq. (7). We then place in the idler path an analyzer that is complementary to the SPP in the signal beam at an orientation $\alpha$; i.e., the SPP is represented by $\hat{S}^{\dagger}(\alpha, j+$ $\left.\frac{1}{2}\right)=\hat{S}\left(\alpha,-j-\frac{1}{2}\right)$. The probability amplitude for both detectors firing in coincidence is given by

$$
B(\alpha)=C\left\langle a_{1 / 2}^{(j)}(\alpha) \mid a_{1 / 2}^{(j)}(0)\right\rangle,
$$

where

$$
C=\frac{1}{\sqrt{2 \pi}}\left(\left\langle\left.\rho_{00}\right|_{1}\left\langle\left.\rho_{00}\right|_{2}\right) \mid R\right\rangle,\right.
$$

and $\alpha \in[0,2 \pi)$. We then find the probability that both detectors fire in coincidence,

$$
|B(\alpha)|^{2}=|C|^{2}\left(1-\frac{\alpha}{\pi}\right)^{2} .
$$

So we find a parabolic coincidence fringe similar to the mode overlap probability in Eq. (3).

The above result is valid for any choice of the signal SPP orientation and depends only on the relative orientation $\alpha$ of the signal and idler SPPs. Thus a coincidence measurement on entangled OAM pairs using half-integer OAM analyzers will bring forth a coincidence fringe that is parabolic, regardless of the individual settings of the analyzers.

A sufficient requirement to prove high-dimensional entanglement of two particles is to demonstrate violation of a generalized Bell inequality. There have been several theoretical papers that address this issue [16] for $\mathrm{N}$ dimensional particles (qunits) [3,17-21]; an example of a qunit is a spin- $s$ particle with $2 s+1=N$. In our case, we do not deal with qunits: each SPP imprints the infinite OAM dimensionality of the (oriented) edge on a transmitted light field. Rotation of this edge is equivalent to a partial exploration of the complete Hilbert space along a certain path, namely, an iso-OAM path; due to this complexity, it is not clear how a generalized Bell inequality could be applied to our case.

However, instead of using a generalized highdimensional bipartite Bell inequality, it is allowed to use an inequality for lower-dimensional two-particle entanglement [21]. Thus we choose, as in the polarization case, the inequality introduced by Clauser, Horne, Shimony, and Holt (CHSH) for a measurement where the coincidence probability is expected to be a function of only $\alpha_{\mathrm{s}}-\alpha_{\mathrm{i}}$ [22]. When relabeling $\alpha_{\mathrm{s}}$ and $\alpha_{\mathrm{i}}$ as $\alpha_{1}$ and $\alpha_{2}$ (in no particular order), the $\mathrm{CHSH}$ inequality is given by $[7,23]$

$S=E\left(\alpha_{1}, \alpha_{2}\right)-E\left(\alpha_{1}^{\prime}, \alpha_{2}\right)+E\left(\alpha_{1}, \alpha_{2}^{\prime}\right)+E\left(\alpha_{1}^{\prime}, \alpha_{2}^{\prime}\right) \leq 2$.

The function $E$ is specified for the variables $x, y$ as $[7,24]$

$$
E(x, y)=\frac{P(x, y)+P\left(x^{\perp}, y^{\perp}\right)-P\left(x, y^{\perp}\right)-P\left(x^{\perp}, y\right)}{P(x, y)+P\left(x^{\perp}, y^{\perp}\right)+P\left(x, y^{\perp}\right)+P\left(x^{\perp}, y\right)} .
$$

The notation $x^{\perp}$ (and similarly for $y^{\perp}$ ) is used to indicate an analyzer setting that analyzes a state orthogonal to the state with setting $x$. Thus in our case, $x^{\perp} \equiv x+\pi$ and $y^{\perp} \equiv y+\pi . P(x, y)$ is the coincidence probability function, which is equal to $P(x, y)=|B(|y-x|)|^{2}$. As the periodicity in the present case is half that of the case of polarization entanglement, we use the standard analyzer settings for polarization entanglement [7,25] multiplied by a factor of 2: $\alpha_{1}=-\frac{1}{4} \pi, \alpha_{1}^{\prime}=\frac{1}{4} \pi, \alpha_{2}=-\frac{1}{2} \pi$, and $\alpha_{2}^{\prime}=0$.

Substitution yields a Bell parameter $S=3 \frac{1}{5}$. This is the key result of our Letter; it indicates that in the case of entanglement of half-integer OAM states the maximum violation of the CHSH inequality, given by Eq. (11), is stronger than the maximum violation that is allowed in polarization entanglement, namely, $S=2 \sqrt{2}$. In other words, quantum nonlocality of the photons in the proposed setup is stronger than the maximum achievable for two qubits. To achieve this, only two detectors are required and only one coincidence count rate is measured per analyzer setting, in contrast to the OAM qunit setup 
requiring $N$ detectors and $N^{2}$ coincidence count rates per analyzer setting $[3,19]$.

In this Letter we have put forward a novel approach to demonstrate high-dimensional entanglement of orbital angular momentum states. The proposed setup uses analyzers that consist of noninteger SPPs and single-mode fibers, enabling detection of high-dimensional entanglement with only two detectors.

The key idea is to use the orientation of the edge dislocation in the SPPs. We specialize to the case of half-integer $\ell$ so that the orientation of the edge as an analyzer setting can, to a certain extent, be treated similarly as the axis of a polarizer in polarization entanglement. We analytically calculate the coincidence fringe in the entanglement setup and find it to be parabolic in shape and periodic over $2 \pi$. When evaluating the well-known CHSH Bell parameter, we find $S=3 \frac{1}{5}$; i.e., we predict beyond-Bell pairing of two photons. This seems to be a consequence of the singular nature of our half-integer SPPs, which implies, in principle, infinite dimensionality. Experimental verification of the outlined proposal is under way.

We acknowledge M. P. van Exter for fruitful discussions regarding the $\mathrm{CHSH}$ version of the Bell inequality. This work is part of the research program of the "Stichting voor Fundamenteel Onderzoek der Materie (FOM)" and is supported by the EU Program ATESIT.

[1] L. Allen, M.W. Beijersbergen, R. J. C. Spreeuw, and J. P. Woerdman, Phys. Rev. A 45, 8185 (1992).

[2] A. Mair, A. Vaziri, G. Weihs, and A. Zeilinger, Nature (London) 412, 313 (2001).

[3] A. Vaziri, G. Weihs, and A. Zeilinger, Phys. Rev. Lett. 89, 240401 (2002).

[4] J. Preskill, Phys. Today 52, 24 (1999).
[5] M.W. Beijersbergen, R. P. C. Coerwinkel, M. Kristensen, and J. P. Woerdman, Opt. Commun. 112, 321 (1994).

[6] Handbook of Mathematical Functions, edited by M. Abramowitz and I. A. Stegun (Dover, New York, 1965).

[7] P. G. Kwiat, K. Mattle, H. Weinfurter, and A. Zeilinger, Phys. Rev. Lett. 75, 4337 (1995).

[8] H. H. Arnaut and G. A. Barbosa, Phys. Rev. Lett. 85, 286 (2000).

[9] E. R. Eliel, S. M. Dutra, G. Nienhuis, and J. P. Woerdman, Phys. Rev. Lett. 86, 5208 (2001).

[10] H. H. Arnaut and G. A. Barbosa, Phys. Rev. Lett. 86, 5209 (2001).

[11] S. Franke-Arnold, S. M. Barnett, M. J. Padgett, and L. Allen, Phys. Rev. A 65, 033823 (2002).

[12] G. A. Barbosa and H. H. Arnaut, Phys. Rev. A 65, 053801 (2002).

[13] J. P. Torres, Y. Deyanova, L. Torner, and G. MolinaTerriza, Phys. Rev. A 67, 052313 (2003).

[14] J. Visser and G. Nienhuis, Eur. Phys. J. D 29, 301 (2004).

[15] S. S. R. Oemrawsingh, A. Aiello, E. R. Eliel, G. Nienhuis, and J. P. Woerdman, quant-ph/0401148.

[16] J. Bell, Physics (Long Island City, N.Y.) 1, 195 (1964).

[17] N. D. Mermin, Phys. Rev. D 22, 356 (1980).

[18] A. Peres, Phys. Rev. A 46, 4413 (1992).

[19] D. Kaszlikowski, P. Gnaciński, M. Żukowski, W. Miklaszewski, and A. Zeilinger, Phys. Rev. Lett. 85, 4418 (2000).

[20] T. Durt, D. Kaszlikowski, and M. Żukowski, Phys. Rev. A 64, 024101 (2001).

[21] D. Collins, N. Gisin, N. Linden, S. Massar, and S. Popescu, Phys. Rev. Lett. 88, 040404 (2002).

[22] J. F. Clauser, M. A. Horne, A. Shimony, and R. A. Holt, Phys. Rev. Lett. 23, 880 (1969).

[23] A. Peres, Quantum Theory: Concepts and Methods (Kluwer Academic Publishers, Dordrecht, 1993).

[24] A. Garuccio and V. A. Rapisarda, Nuovo Cimento Soc. Ital. Fis. 65A, 269 (1981).

[25] J. F. Clauser and M. A. Horne, Phys. Rev. D 10, 526 (1974). 